\title{
A HYBRID METHOD FOR ACCELERATED SIMULATION OF COULOMB COLLISIONS IN A PLASMA*
}

\author{
RUSSEL CAFLISCH ${ }^{\dagger}$, CHIAMING WANG ${ }^{\dagger}$, GIACOMO DIMARCO $^{\ddagger}$, BRUCE COHEN $^{\S}$, \\ AND ANDRIS DIMITS ${ }^{\S}$
}

\begin{abstract}
If the collisional time scale for Coulomb collisions is comparable to the characteristic time scales for a plasma, then simulation of Coulomb collisions may be important for computation of kinetic plasma dynamics. This can be a computational bottleneck because of the large number of simulated particles and collisions (or phase-space resolution requirements in continuum algorithms), as well as the wide range of collision rates over the velocity distribution function. This paper considers Monte Carlo simulation of Coulomb collisions using the binary collision models of Takizuka and Abe and of Nanbu. It presents a hybrid method for accelerating the computation of Coulomb collisions. The hybrid method represents the velocity distribution function as a combination of a thermal component (a Maxwellian distribution) and a kinetic component (a set of discrete particles). Collisions between particles from the thermal component preserve the Maxwellian; collisions between particles from the kinetic component are performed using the method of Takizuka and Abe or of Nanbu. Collisions between the kinetic and thermal components are performed by sampling a particle from the thermal component and selecting a particle from the kinetic component. Particles are also transferred between the two components according to thermalization and dethermalization probabilities, which are functions of phase space.
\end{abstract} tion

Key words. Coulomb collisions, plasma, simulation, hybrid method, bump on tail, thermaliza-

AMS subject classifications. 82D10, 65C05, 76X05

DOI. $10.1137 / 070704939$

1. Introduction. In many plasma systems, the principal interactions between charged particles are Coulombic. For interparticle distance $d$ larger than the Debye length $\lambda_{D}$, Coulomb interactions are mediated through electromagnetic fields governed by a Vlasov equation. On the other hand, if $d<\lambda_{D}$, these interactions can be described as Coulomb collisions, governed by the Fokker-Planck equation.

The Fokker-Planck equation has a time scale $t_{F P}$, defined by the rate of change of the particle velocity vector angle. If the characteristic time $t_{0}$ of interest is large compared to $t_{F P}$, then Coulomb interactions will drive the velocity distribution $f(\mathbf{v})$ to its equilibrium, given by a Maxwellian distribution $M$, with density $n_{M}$, velocity $\mathbf{u}_{M}$, and temperature $T_{M}$. Further evolution of the system can be described by continuum equations for $n_{M}, \mathbf{u}_{M}$, and $T_{M}$. At the other extreme if $t_{0}<<t_{F P}$, the plasma can be described as collisionless. In the intermediate regime with $t_{0}$ and $t_{F P}$

${ }^{*}$ Received by the editors October 9, 2007; accepted for publication (in revised form) May 5, 2008; published electronically August 20, 2008. This work was performed under the auspices of the U.S. DOE by the University of California, Los Angeles, under grant DE-FG02-05ER25710, and by Lawrence Livermore National Laboratory under contract DE-AC52-07NA27344. This work was supported by the Office of Advanced Scientific Computing Research, DOE Office of Science, under the Multiscale Initiative Program. The U.S. Government retains a nonexclusive, royalty-free license to publish or reproduce the published form in this contribution, or allow others to do so, for U.S. Government purposes. Copyright is owned by SIAM to the extent not limited by these rights.

http://www.siam.org/journals/mms/7-2/70493.html

${ }^{\dagger}$ Mathematics Department, University of California at Los Angeles, Los Angeles, CA 90095 (caflisch@math.ucla.edu, cmwang@math.ucla.edu).

${ }^{\ddagger}$ Mathematics Department, University of Ferrara, Ferrara 44100, Italy (dmrgcm@unife.it).

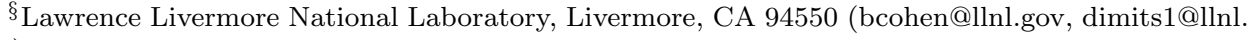
gov). 
of comparable size, the kinetics of Coulomb collisions are significant for the evolution of the velocity distribution function for the plasma.

This paper is concerned with Monte Carlo particle methods for simulation of Coulomb collisions in a plasma using binary collisions. One of the earliest and most influential Monte Carlo binary collision models was proposed by Takizuka and Abe (TA) in 1977 [14] and modified by Nanbu in 1997 [9]. In a subsequent work, Bobylev and Nanbu [2] derived a general time-explicit formulation for the approximation of the Fokker-Planck equation by a binary collision model. Wang et al. [16] performed a numerical convergence study for the methods of TA and Nanbu.

The two methods proposed by TA [14] and Nanbu [9] have been widely used in the plasma physics community. Simulation of Coulomb collisions can be a computational bottleneck, however, since the collision times are often very different from the characteristic times of interest. This difficulty is compounded by the wide range of collision rates for many problems. For example, consider a velocity distribution in the form of a bump on tail, i.e., a near-equilibrium distribution at low velocity with an isolated spike far out on its tail (the "bump"). The rate of collisions between two particles of velocity $\mathbf{v}_{1}$ and $\mathbf{v}_{2}$ is proportional to $u^{-3}$ for $u=\left|\mathbf{v}_{1}-\mathbf{v}_{2}\right|$. The average rate of collisions between the particles in the central distribution $f \approx M$ is of size $T_{M}^{-3 / 2}$, in which $T_{M}$ is the temperature of the Maxwellian distribution $M$. The bump may be concentrated at a velocity difference $u_{B}$ from the center of $M$ with $u_{B} \gg T_{M}^{1 / 2}$, so that its rate of interaction with $M$ is of size $u_{B}^{-3}<<T_{M}^{-3 / 2}$. Direct simulation of the Coulomb collisions for a bump-on-tail distribution is dominated by collisions between $M$ and itself, which preserve $M$ but do not affect the evolution of $f$, and the important interactions of the bump with $M$ will be rare events. This shows that direct simulation of this problem is highly inefficient.

The purpose of this paper is to present a hybrid method for accelerating the simulation of Coulomb collisions. It represents the distribution function as a combination of a thermal component $m$ (a Maxwellian distribution) and kinetic component $k$ (numerically represented as a set of particles). Evolution of the thermal component $m$ is performed using continuum methods based on conservation principles, while evolution of the kinetic component $k$ is performed by Monte Carlo simulation of binary collisions using the method of TA or Nanbu. An interaction between $m$ and $k$ is performed by sampling a particle from $m$ and selecting a particle from $k$ and then treating the interaction as a particle collision. In addition, thermalization (particles moved from $k$ to $m$ ) and dethermalization (particles moved from $m$ to $k$ ) are performed with probabilities $p_{T}$ and $p_{D}$, respectively. In this approach, the thermalization/dethermalization step is considered to be a part of the reorganization of the velocity distribution into the form of a Maxwellian plus a perturbation, rather than being a part of the collision process.

Although the physical significance of the hybrid method is fairly transparent, we do not have a full mathematical derivation for it. In particular, the relation between the thermalization/dethermalization and the collision process is not mathematically clear. On the other hand, we have a derivation of an equilibrium requirement - similar to the detailed balance condition - for the thermalization and dethermalization probabilities. In addition, the computational results presented in this paper demonstrate the consistency and accuracy of the method, as well as the character of the numerical solutions. The method is not yet complete, because it does not yet include spatial transport of the charged particles, and our implementation is not optimized.

This hybrid method is motivated by a similar hybrid method for rarefied gas dynamics (RGD) developed by Pareschi and Caflisch [10], which combines the direct 
simulation Monte Carlo (DSMC) method [1] with a fluid dynamic solver. There are several significant differences between the hybrid method for RGD and that for Coulomb collisions. For RGD, there is a mathematical derivation of the thermalization and dethermalization: Particles that experience a large number of collisions in a single time step are thermalized, while the portion of the Maxwellian component that collides with the particle component is dethermalized. The collision rates have a much wider range of values for Coulomb collisions than for RGD, because the Coulomb collision rate varies strongly in phase space (i.e., $\mathbf{x}$ and $\mathbf{v}$ ), whereas the RGD collision rate varies mainly in physical space (i.e., $\mathbf{x}$ only). As a result, the division between Maxwellian and particle components is performed solely in physical space $\mathbf{x}$ for RGD. For Coulomb collisions, however, the division between the two components must be performed in phase space $(\mathbf{x}, \mathbf{v})$, and $p_{T}$ and $p_{D}$ are functions of $\mathbf{x}$ and $\mathbf{v}$. The relations between the DSMC method and the methods of TA and Nanbu are discussed further in section 2 .

The hybrid method presented here has the potential to accelerate kinetic computations for Coulomb collisions in a plasma. Currently, simulations that use the full distribution function ("full $f$ " methods) are often prohibitively expensive computationally. Alternative acceleration methods (" $\delta f$ " methods) $[3,4,5,6]$ represent the velocity distribution function as $f=M+\delta f$ and solve linearized (or partially linearized) equations for $\delta f$. These methods have been successful in many applications but are not applicable in some important regimes such as the edge region of a magnetically confined fusion plasma. Our aim is to overcome the computational difficulties of the full $f$ methods for problems that cannot be addressed with $\delta f$ methods.

The remainder of this paper is organized as follows: The Monte Carlo binary collision methods of TA [14] and Nanbu [9], as well as the general formulation of Bobylev and Nanbu [2], are presented in section 2, and the hybrid method is formulated in section 3. Determination of $p_{T}$ and $p_{D}$ is performed in section 4 through a detailed balance requirement and the use of Nanbu's $s$ parameter. Computational results are presented in section 5. These include a series of computations to illuminate the character of the solutions of the hybrid method, followed by a study of the accuracy and efficiency of the method. Conclusions and a discussion of further research directions are presented in section 6 .

2. Monte Carlo simulation of Coulomb collisions. We first introduce the governing equation for the physical process and describe the TA and Nanbu Monte Carlo binary collision models for a spatially homogeneous plasma. We consider collisions between particles of two species $\alpha$ and $\beta$ and describe simulations that involve $N$ particles consisting of $N / 2$ particles from each of the species. Spatial dependence and electromagnetic fields are omitted.

For the two species, denote $v_{\alpha}$ and $v_{\beta}$ to be the particle velocities, $f_{\alpha}$ and $f_{\beta}$ to be the velocity distribution functions, $m_{\alpha}$ and $m_{\beta}$ to be the particle masses, $n_{\alpha}$ and $n_{\beta}$ to be the particle number densities, and $q_{\alpha}$ and $q_{\beta}$ to be the particle charges. In a later section, there is only a single species, and we will use the same notation without any subscript. Also define the free space electrical permittivity $\varepsilon_{0}$ and the reduced mass $m_{\alpha \beta}=m_{\alpha} m_{\beta} /\left(m_{\alpha}+m_{\beta}\right)$. The Fokker-Planck time scale (e.g., in [15]) is defined by (in MKS units)

$$
t_{F P}=\left(\frac{c_{F P} n_{L}}{u_{\alpha \beta}^{3}}\right)^{-1}
$$




$$
c_{F P}=\frac{q_{\alpha}^{2} q_{\beta}^{2}}{4 \pi \epsilon_{0}^{2} m_{\alpha \beta}^{2}} \log \Lambda,
$$

in which $n_{L}=\min \left(n_{\alpha}, n_{\beta}\right)$, and $u_{\alpha \beta}$ is the root mean square of the difference between the velocity of an $\alpha$ and that of a $\beta$ particle. The Coulomb logarithm $\log \Lambda$ and the Debye length $\lambda_{D}$ come from the cutoff of the Coulomb potential at a large distance and are defined by

$$
\begin{array}{r}
\Lambda=\left(2 \pi \varepsilon_{0} T m_{\alpha \beta} \lambda_{D}\right) /\left|q_{\alpha} q_{\beta}\right|, \\
\lambda_{D}=\left(n q^{2} / \varepsilon_{0} T\right)^{-1 / 2},
\end{array}
$$

in which $T$ is temperature in units of energy (i.e., the Boltzmann constant $k$ is included in $T$ ). In numerical simulations, $\Lambda$ is often replaced by a numerical parameter that is taken to be independent of the physical parameters.

2.1. Fokker-Planck equation. Coulomb interactions between charged particles are long range with a potential decaying at a rate $r^{-1}$ for interparticle distance $r$. In a plasma with quasi neutrality of the charges, Debye shielding [7] reduces the potential to $r^{-1} e^{-r / \lambda_{D}}$. Interactions over distance $r>\lambda_{D}$ are included through electric and magnetic fields, while those for $r<\lambda_{D}$ are described by direct interactions between particles. Typical plasmas contain many particles within a Debye volume $\lambda_{D}^{3}$, and the resulting Coulomb interactions can be thought of as a large number of small-angle binary collisions [13].

The resulting time evolution of the particle distribution in a spatially homogeneous, nonequilibrium plasma is described by drag and diffusion in velocity space through the Fokker-Planck equation

$$
\frac{\partial f_{\alpha}}{\partial t}=\sum_{\beta}\left\{-\frac{\partial}{\partial \mathbf{v}} \cdot \mathbf{F}_{d}(\mathbf{v}) f(\mathbf{v})+\frac{1}{2} \frac{\partial^{2}}{\partial \mathbf{v} \partial \mathbf{v}}: \mathbf{D}(\mathbf{v}) f(\mathbf{v})\right\}
$$

in which

$$
\begin{aligned}
\mathbf{F}_{d}(\mathbf{v}) & =c_{F P} \frac{m_{\alpha \beta}}{m_{\alpha}} \frac{\partial H}{\partial \mathbf{v}}=c_{F P} \frac{m_{\alpha \beta}}{m_{\alpha}} \frac{\partial}{\partial \mathbf{v}} \int \frac{f_{\beta}\left(\mathbf{v}^{\prime}\right)}{\left|\mathbf{v}-\mathbf{v}^{\prime}\right|} d \mathbf{v}^{\prime} \\
\mathbf{D}(\mathbf{v}) & =c_{F P} \frac{m_{\alpha \beta}^{2}}{m_{\alpha}^{2}} \frac{\partial^{2} G}{\partial \mathbf{v} \partial \mathbf{v}}=c_{F P} \frac{m_{\alpha \beta}^{2}}{m_{\alpha}^{2}} \frac{\partial^{2}}{\partial \mathbf{v} \partial \mathbf{v}} \int f_{\beta}\left(\mathbf{v}^{\prime}\right)\left|\mathbf{v}-\mathbf{v}^{\prime}\right| d \mathbf{v}^{\prime} .
\end{aligned}
$$

The functions $\mathbf{F}_{d}$ and $\mathbf{D}$ are the drift vector and diffusion matrix, and $H$ and $G$ are the Rosenbluth potentials [11]. Since the drift has the form $\mathbf{F}_{d}(\mathbf{v})=F_{d}(v)(\mathbf{v} / v)$, it can be thought of as a drag term.

The Fokker-Planck equation (3) can be rewritten in the following Landau form:

$$
\frac{\partial f_{\alpha}}{\partial t}=-\sum_{\beta} \frac{c_{F P} m_{\alpha \beta}^{2}}{2 m_{\alpha}} \frac{\partial}{\partial v_{\alpha j}} \int d \mathbf{v}_{\beta}\left[\frac{\delta_{j k}}{u}-\frac{u_{j} u_{k}}{u^{3}}\right]\left[\frac{f_{\alpha}}{m_{\beta}} \frac{\partial f_{\beta}}{\partial v_{\beta k}}-\frac{f_{\beta}}{m_{\alpha}} \frac{\partial f_{\alpha}}{\partial v_{\alpha k}}\right],
$$

in which we use the notation $\mathbf{u}=\mathbf{v}_{\alpha}-\mathbf{v}_{\beta}, u=|\mathbf{u}|, f_{\alpha}=f_{\alpha}\left(\mathbf{v}_{\alpha}\right)$, and $f_{\beta}=f_{\beta}\left(\mathbf{v}_{\beta}\right)$, as well as the Einstein summation convention. The equation for $f_{\beta}$ is similar.

Because of the high dimensionality of the Fokker-Planck equation (3) or (5), the most efficient simulation method is often a Monte Carlo method. There are two different types of Monte Carlo methods used to solve (3) and (5). The first is a Monte 
Carlo method using a drag/diffusion formulation developed by Mannheimer, Lampe, and Joyce [8] and extended recently in [12]. In their method each particle velocity evolves due to drift (drag) and random jumps (diffusion), corresponding to the drift velocity $\mathbf{F}_{d}$ (or drag coefficient $F_{d}$ ) and the diffusion coefficient $\mathbf{D}$, as in (3). The particle velocities (and positions) are then used to determine $\mathbf{F}_{d}$ and $\mathbf{D}$, as in (4).

The second Monte Carlo method using a binary collision formulation was developed by TA [14]. They interpreted the terms in (5) as binary collisions, in the spirit of the DSMC method developed by Bird [1] for RGD. In both of these methods, the number of numerical particles is substantially less than the physical number of particles, and the numerical collisions are performed randomly in order to represent the much larger number of physical collisions. Significant differences between the TA method for Coulomb collisions and Bird's DSMC method are in the rate and meaning of the numerical collisions. In the usual DSMC method, each numerical collision represents a real collision between two gas particles, and each numerical particle experiences the physically correct number of collisions in a time step. Because of the very large rate of Coulomb interactions, in the TA, Nanbu, and Bobylev-Nanbu methods, each collision represents an aggregate of many small-angle interactions. Moreover, each numerical particle undergoes a single collision in each time step $\Delta t$; and the scattering angle for each numerical collision depends on $\Delta t$.

2.2. Bobylev-Nanbu approach. Bobylev and Nanbu [2] developed a general formulation for a binary collision model that approximates the solution of (5) over a time step $\Delta t$. They used this to show that Nanbu's method [9] is an approximation of the Fokker-Planck equation (5). In Appendix A, we apply their analysis to show that the TA method is also an approximation of the Fokker-Planck equation (5).

The binary collision model of Bobylev and Nanbu (see [2] for further details and definitions) is

$$
f_{\alpha}\left(\mathbf{v}_{\alpha}, t+\Delta t\right)=\sum_{\beta=1}^{n} \pi_{\alpha \beta} \int_{R^{3} \times S^{2}} d \mathbf{v}_{\beta} d \mathbf{n} D_{\alpha \beta}\left(\frac{\mathbf{u} \cdot \mathbf{n}}{u}, A_{\alpha \beta} \frac{\Delta t}{u^{3}}\right) f_{\alpha}\left(\mathbf{v}_{\alpha}^{\prime}, t\right) f_{\beta}\left(\mathbf{v}_{\beta}^{\prime}, t\right)
$$

in which $\pi_{\alpha \beta}$ defines the proportion of $\alpha$ particles that have a collision with $\beta$ particles, $A_{\alpha \beta}$ is a rate factor for collisions between $\alpha$ and $\beta$ particles, and $\mathbf{n}$ is the normal vector on the sphere at a point with angular coordinates $(\theta, \psi)$, which are the scattering angles. For velocities $\mathbf{v}_{\alpha}$ and $\mathbf{v}_{\beta}$ after a collision, $\mathbf{v}_{\alpha}^{\prime}$ and $\mathbf{v}_{\beta}^{\prime}$ are the velocities before the collision (or vice versa, since collisions are reversible), given by

$$
\begin{aligned}
& \mathbf{v}_{\alpha}^{\prime}=\mathbf{v}_{\alpha}+\frac{m_{\alpha \beta}}{m_{\alpha}} \Delta \mathbf{u}, \\
& \mathbf{v}_{\beta}^{\prime}=\mathbf{v}_{\beta}-\frac{m_{\alpha \beta}}{m_{\beta}} \Delta \mathbf{u},
\end{aligned}
$$

in which

$$
\begin{aligned}
\Delta u_{x} & =\left(u_{x} / u_{\perp}\right) u_{z} \sin \theta \cos \phi-\left(u_{y} / u_{\perp}\right) u \sin \theta \sin \phi-u_{x}(1-\cos \theta), \\
\Delta u_{y} & =\left(u_{y} / u_{\perp}\right) u_{z} \sin \theta \cos \phi+\left(u_{x} / u_{\perp}\right) u \sin \theta \sin \phi-u_{y}(1-\cos \theta), \\
\Delta u_{z} & =-u_{\perp} \sin \theta \cos \phi-u_{z}(1-\cos \theta), \\
\mathbf{u} & =\mathbf{v}_{\alpha}-\mathbf{v}_{\beta}, \\
u_{\perp} & =\sqrt{u_{x}^{2}+u_{y}^{2}} .
\end{aligned}
$$


The interpretation of this equation by Bobylev and Nanbu is that in a single time step, each particle with velocity $v_{\alpha}^{\prime}$ undergoes a collision with some particle with velocity $v_{\beta}^{\prime}$ and is replaced by the resulting particle with velocity $v_{\alpha}$. The rate of numerical collisions is independent of the particle velocities, so that the particles for a collision can be chosen randomly (independent of their velocity), as in RGD for Maxwell molecules. The kernel $2 \pi D$ describes the probability density for the choice of scattering angle; since $2 \pi \int_{-1}^{1} d \mu D(\mu, \tau)=1$, then $\mu=\cos \theta$ is chosen uniformly on the interval $[-1,1]$. One advantage of this interpretation is that virtual collisions (i.e., trial collisions that must be randomly accepted or rejected) and Bird's no-time counter method are not required for simulation of Coulomb collisions using the TA or Nanbu methods or any method that follows the approach of Bobylev and Nanbu.

Nanbu [9] defined a parameter $s$ that describes the mean-square deflection of a scattered particle as a function of the time step $\Delta t$ and the relative velocity $u$ between two colliding particles. To simplify the comparison of our equations with those in [2] and [9], we use parameters $\tau$ from [2] and $s$ from [9], defined by

$$
\tau=\frac{s}{2}=\frac{n_{L}}{t_{F P} u^{3}} \Delta t
$$

They also found a set of conditions (written out in Appendix A) on the kernel $D_{\alpha \beta}$ which ensure that $f$ is an approximate solution of (5) with first order accuracy. As described in the following, the TA and Nanbu collision models each correspond to a Monte Carlo simulation of the integral (6) for a specific choice of $D_{\alpha \beta}$.

2.3. The collision model of TA. Although the TA model was not analyzed in [2], we show that it fits into the Bobylev-Nanbu formulation. In particular, we show that the collision model of TA corresponds to the following formula for $D=D_{T A}$ :

$$
D_{T A}(\mu, \tau)=(2 \pi)^{-1}(2 \pi \tau)^{-1 / 2} e^{-\zeta^{2} / 2 \tau}(d \zeta / d \mu) .
$$

The scattering angle $\theta$ in the frame of the relative velocity $\mathbf{u}$ is defined by

$$
\begin{aligned}
\theta & =2 \arctan \zeta, \\
\mu & =\cos \theta .
\end{aligned}
$$

With the choice $D=D_{T A}$, the convergence criteria of Bobylev and Nanbu in [2] are satisfied, as shown in Appendix A.

A Monte Carlo algorithm for simulation of the integral (6) with the kernel (10) over a single time interval $\Delta t$ consists of performing the following steps $N / 2$ times:

1. Randomly select two particles with velocities $\mathbf{v}_{\alpha}$ and $\mathbf{v}_{\beta}$ from the distributions $f_{\alpha}$ and $f_{\beta}$. This is done by exclusive sampling, so that no particle is selected more than once. This corresponds to the term $f_{\alpha} f_{\beta}$ in (6).

2. Sample a value of $\mu=\cos (2 \arctan \zeta)$, in which $\zeta$ is a Gaussian random variable with mean 0 and variance $\tau=\left\langle\zeta^{2}\right\rangle$ and $\tau$ is defined by (9) using $u=\left|\mathbf{v}_{\alpha}-\mathbf{v}_{\beta}\right|$. Define $\theta$ by $\theta=2 \arctan \zeta$. This corresponds to the factor $(2 \pi \tau)^{-1 / 2} e^{-\zeta^{2} / 2 \tau}(d \zeta / d \mu)$ in $D_{T A}$.

3. Choose the azimuthal angle $\phi$ randomly and uniformly from the interval $[0,2 \pi]$. This corresponds to the remaining factor $(2 \pi)^{-1}$ in $D_{T A}$.

4. The new velocities are $\mathbf{v}_{\alpha}^{\prime}$ and $\mathbf{v}_{\beta}^{\prime}$ defined by $(7)$.

5. Replace the velocities $\mathbf{v}_{\alpha}$ and $\mathbf{v}_{\beta}$ by $\mathbf{v}_{\alpha}^{\prime}$ and $\mathbf{v}_{\beta}^{\prime}$. This corresponds to the appearance of $\mathbf{v}_{\alpha}^{\prime}$ and $\mathbf{v}_{\beta}^{\prime}$ as the arguments of $f_{\alpha}$ and $f_{\beta}$ in (6). 
These are exactly the steps of the algorithm described in the work of TA [14]. Note that in this algorithm, as well as in the algorithm of Nanbu and the general formulation of [2], every particle collides exactly once in each time interval.

2.4. Nanbu's collision model. Nanbu's method is based on a parameter $s$ that measures the average angular deflection of a particle over a time step $\Delta t$. As described in [2], the collision model of Nanbu corresponds to the following formula for $D=D_{N a n b u}$ :

$$
D_{N a n b u}(\mu, \tau)=\frac{A}{4 \pi \sinh A} \exp \mu A,
$$

in which $A$ is defined by

$$
\operatorname{coth} A-A^{-1}=e^{-s}=e^{-2 \tau} .
$$

Monte Carlo simulation using this kernel over a single time interval $\Delta t$ is exactly the same as the simulation for TA given above, except that step 2 is replaced by the following:

2'. Calculate the quantities $s$ and $A$ solving (13) using $u=\left|\mathbf{v}_{\alpha}-\mathbf{v}_{\beta}\right|$ in the definition (9) of $\tau$. Sample a value of the random variable $\mu$ from the interval $[-1,1]$ with probability density

$$
f(\mu)=2 \pi D_{N a n b u}=A(2 \sinh A)^{-1} e^{A \mu}
$$

and define $\theta$ by $\mu=\cos (\theta)$.

These are exactly the steps of the algorithm described in the work of Nanbu [9] with some minor changes in notation for consistency with the TA method.

In the remainder of the paper, the collisions are assumed to be between particles from a single species, so that the subscripts $\alpha$ and $\beta$ are dropped. In addition, the distribution function will be assumed to be spatially homogeneous, so that particle position can be neglected.

3. The hybrid method. In formulating a hybrid method, we hope to take advantage of two multiscale features for Coulomb collisions. The first is the separation between the fluid dynamic time scale and the collisional time scale for problems in which the collisional time scale is relatively small (i.e., a small Knudsen number) but not so small that a continuum description is justified. In this case, separation of the velocity distribution function $f$ into a Maxwellian component and a remainder allows use of fluid dynamics for efficient simulation of the Maxwellian component.

The second multiscale feature is the large variation of the scattering rate over phase space. For problems such as a bump-on-tail distribution there is a wide separation between different features in phase space, e.g., between the central Maxwellian and the bump. In this case, the fast interactions of the particles within the Maxwellian do not require simulation, since they leave the Maxwellian unchanged. A larger fraction of the computational effort can then be applied to the slow interactions between particles in the Maxwellian and those in the bump.

The hybrid method is based on representation of the velocity distribution function $f$ as a combination of a thermal component $m$ and a kinetic component $k$; i.e.,

$$
f(\mathbf{v})=m(\mathbf{v})+k(\mathbf{v}) .
$$

The thermal component is a Maxwellian distribution

$$
m(\mathbf{v})=n_{m}\left(2 \pi T_{m}\right)^{-3 / 2} \exp \left(-\left|\mathbf{v}-\mathbf{u}_{m}\right|^{2} / 2 T_{m}\right) .
$$


Because of the (expected) slow interaction of the thermal component $m$ with the kinetic component $k$, the average density, velocity, and temperature $n_{m}, \mathbf{u}_{m}$, and $T_{m}$ of $m$ are not assumed to be those of the full distribution $f$. This explains the difference between the notation $m$ and $M$, since $M$ is assumed to have density, velocity, and temperature that are equal to those of $f$.

Denote $n_{m}$ and $n_{k}$ to be the effective number of particles in the thermal and kinetic components, respectively, of $f$. At present these numbers will be kept to be even integers. The kinetic component will be simulated using a set of discrete particles; i.e.,

$$
k(\mathbf{v})=\sum_{i=1}^{n_{k}} \delta\left(\mathbf{v}-\mathbf{v}_{i}\right)
$$

In each time interval, the simulation steps are the following:

1. Determine the number of collisions of each type, so that each simulation particle experiences only one collision of any kind when collisions are computed.

- $n_{m m}=n_{m}^{2} / 2\left(n_{k}+n_{m}\right)$ is the number of collisions between $2 m$ particles.

- $n_{k k}=n_{k}^{2} / 2\left(n_{k}+n_{m}\right)$ is the number of collisions between $2 k$ particles.

- $n_{m k}=n_{m} n_{k} /\left(n_{k}+n_{m}\right)$ is the number of collisions between an $m$ particle and a $k$ particle.

2. Perform the collisions.

- The $m-m$ collisions do not change the distribution $m$, so they do not need to be performed.

- For each $k-k$ collision, select two particles $\mathbf{v}_{k 1}$ and $\mathbf{v}_{k 2}$ from $k$. Perform a collision between them, as in the method of TA or Nanbu, to get new velocities $\mathbf{v}_{k 1}^{\prime}$ and $\mathbf{v}_{k 2}^{\prime}$. In $k$, replace $\mathbf{v}_{k 1}$ and $\mathbf{v}_{k 2}$ by $\mathbf{v}_{k 1}^{\prime}$ and $\mathbf{v}_{k 2}^{\prime}$.

- For each $m-k$ collision, sample a particle $\mathbf{v}_{m}$ from $m$ and select a particle $\mathbf{v}_{k}$ from $k$. Perform a collision between them, as in the method of TA or Nanbu, to get new velocities $\mathbf{v}_{m}^{\prime}$ and $\mathbf{v}_{k}^{\prime}$. The postcollision velocity $\mathbf{v}_{k}^{\prime}$ replaces $\mathbf{v}_{k}$ in $k$.

3. Apply thermalization and dethermalization.

- For each postcollision particle $\mathbf{v}^{\prime}$ from the kinetic component (i.e., $\mathbf{v}_{k 1}^{\prime}$, $\mathbf{v}_{k 2}^{\prime}$, or $\mathbf{v}_{k}^{\prime}$ from the previous step), thermalize $\mathbf{v}^{\prime}$ with probability $p_{T}\left(\mathbf{v}^{\prime}\right)$. This is done by removing $\mathbf{v}^{\prime}$ from $k$ (in the next step its number, momentum, and energy will be added to $m$ ).

- For each postcollision particle $\mathbf{v}_{m}^{\prime}$, dethermalize $\mathbf{v}_{m}^{\prime}$ with probability $p_{D}\left(\mathbf{v}_{m}^{\prime}\right)$. This is done by adding $\mathbf{v}_{m}^{\prime}$ to $k$ (in the next step its number, momentum, and energy will be subtracted from $m$ ).

4. Apply conservation.

- Adjust the number $n_{k}$ of particles in $k$ due to thermalization and dethermalization.

- Adjust the number, momentum, and energy of $m$ due to thermalization and dethermalization. This is most easily performed by requiring that the total number, momentum, and energy of $f=m+k$ be the same before and after the collisions.

A possible problem with this algorithm is that sampling velocities $\mathbf{v}_{m}$ from $m$ may remove too much energy from $m$. This can be avoided by conservative sampling. First sample all $n_{m k}$ velocities from $m$ and then shift and scale these, so that the average momentum and energy of the sampled particles are the same as the average momentum and energy of $m$. If $\tilde{\mathbf{v}}_{i}$ for $1 \leq i \leq n_{m k}$ are the velocities sampled directly 
from $m$, then the resulting velocities from conservative sampling are $\mathbf{v}_{i}$,

$$
\mathbf{v}_{i}=c\left(\tilde{\mathbf{v}}_{i}-\overline{\mathbf{w}}\right)
$$

in which

$$
\begin{aligned}
\overline{\mathbf{w}} & =\overline{\mathbf{v}}_{e}-c^{-1} \mathbf{u}_{m}, \\
c^{2} & =T_{m} / T_{e} \\
\overline{\mathbf{v}}_{e} & =n_{m k}^{-1} \sum_{i=1}^{n_{m k}} \tilde{\mathbf{v}}_{i}, \\
T_{e} & =n_{m k}^{-1} \sum_{i=1}^{n_{m k}}\left(\tilde{\mathbf{v}}_{i}-\overline{\mathbf{v}}_{e}\right)^{2},
\end{aligned}
$$

and $\mathbf{u}_{m}$ and $T_{m}$ are the average velocity and temperature of the Maxwellian $m$.

\section{Choice of $p_{D}$ and $p_{T}$.}

4.1. Detailed balance condition. We first derive a consistency condition on $p_{D}$ and $p_{T}$ and then present a detailed choice for their dependence on $v$.

Consider an equilibrium distribution $M$ represented as

$$
M=m+k=f,
$$

in which $m$ is the continuum component and $k$ is the kinetic component. Note that $m$ is an equilibrium, but $m$ is not necessarily equal to $M$. In Appendix B, detailed balance is used to derive conditions on $p_{D}$ and $p_{T}$, starting from the scattering integral of (6) with the inclusion of thermalization/dethermalization. Although this is the theoretically correct approach, it does not lead to explicit conditions on $p_{D}$ and $p_{T}$.

In this section, we adopt a simpler approach by requiring that thermalization/ dethermalization applied to all of $f=m+k$ does not change $m$ and $k$ if $f=M$ is a Maxwellian. This is performed as follows: Apply thermalization to $k$ with probability $p_{T}$ and dethermalization to $m$ with probability $p_{D}$. Also define a projection $\Pi_{M}$ onto equilibria; i.e., $\Pi_{M} f$ is the Maxwellian with same $(\rho, \mathbf{u}, T)$ as $f$. The resulting distribution is

$$
f^{\prime}=\Pi_{M}\left(\left(1-p_{D}\right) m+p_{T} k\right)+p_{D} m+\left(1-p_{T}\right) k .
$$

Note that since the projection $\Pi_{M}$ conserves mass, momentum, and energy, the distributions $f$ and $f^{\prime}$ have the same mass, momentum, and energy. Now assume that $f=m+k=M$ and require that the form of $f$ be conserved; i.e.,

$$
\begin{aligned}
m & =\Pi_{M}\left(\left(1-p_{D}\right) m+p_{T} k\right), \\
k & =p_{D} m+\left(1-p_{T}\right) k .
\end{aligned}
$$

It follows that

$$
\begin{aligned}
k & =\left(p_{D} / p_{T}\right) m, \\
M & =\left(1+p_{D} / p_{T}\right) m .
\end{aligned}
$$

Denote

$$
\gamma=M / m
$$


For simplicity assume that

$$
\mathbf{u}_{M}=\mathbf{u}_{m}=0
$$

or, more generally, that $\mathbf{u}_{M}=\mathbf{u}_{m}$. This is not generally true but is a reasonable assumption if $f=M$. Then look for

$$
\gamma(\mathbf{v})=c e^{|\mathbf{v}|^{2} / 2 \tau}
$$

in which

$$
\begin{aligned}
c & =\left(n_{M} / n_{m}\right)\left(T_{m} / T_{M}\right)^{3 / 2}, \\
\tau^{-1} & =T_{m}^{-1}-T_{M}^{-1} .
\end{aligned}
$$

Note that $m<M$ for all $\mathbf{v}$, so that $T_{m}<T_{M}$. Insertion of (26) into (23) shows that the detailed balance requirement for $p_{D}$ and $p_{T}$ is

$$
1+p_{D} / p_{T}=c e^{|\mathbf{v}|^{2} / 2 \tau} .
$$

4.2. Velocity-based choice of $p_{D}$ and $p_{T}$. Now we make a specific choice of the remaining degrees of freedom in $p_{D}$ and $p_{T}$. We expect more thermalization for small $|v|$ and more dethermalization for larger $|v|$. Look for $p_{T}, p_{D}$ to satisfy

$$
\begin{aligned}
& p_{T}=1 \text { for }|\mathbf{v}|<v_{1}, \\
& p_{D}=1 \text { for }|\mathbf{v}|>v_{2},
\end{aligned}
$$

in which $v_{1}$ and $v_{2}$ are constants with $v_{1}<v_{2}$. Define

$$
\begin{aligned}
p_{D} & =\sqrt{\alpha(\gamma-1)}, \\
p_{T} & =\sqrt{\alpha /(\gamma-1)},
\end{aligned}
$$

which automatically satisfy (29). For a given choice of $v_{1}, v_{2}$, set

$$
\begin{aligned}
c & =1, \\
\tau & =(4 \log 2)^{-1}\left(v_{1}^{2}+v_{2}^{2}\right), \\
\gamma_{1} & =\gamma\left(v_{1}\right)=e^{v_{1}^{2} / 2 \tau}, \\
\gamma_{2} & =\gamma\left(v_{2}\right)=e^{v_{2}^{2} / 2 \tau}, \\
\alpha_{1} & =\alpha\left(v_{1}\right)=\left(\gamma_{1}-1\right), \\
\alpha_{2} & =\alpha\left(v_{2}\right)=\left(\gamma_{2}-1\right)^{-1} .
\end{aligned}
$$

The choice of $\tau$ was made so that

$$
0<\alpha_{1}<1, \quad 0<\alpha_{2}<1,
$$

i.e.,

$$
v_{1}^{2} / 2 \tau<\log 2<v_{2}^{2} / 2 \tau .
$$

Since $p_{D}\left(v_{1}\right)=\alpha_{1}$ and $p_{T}\left(v_{2}\right)=\alpha_{2}$, the construction below will ensure that $0 \leq p_{D} \leq$ 1 and $0 \leq p_{T} \leq 1$. 


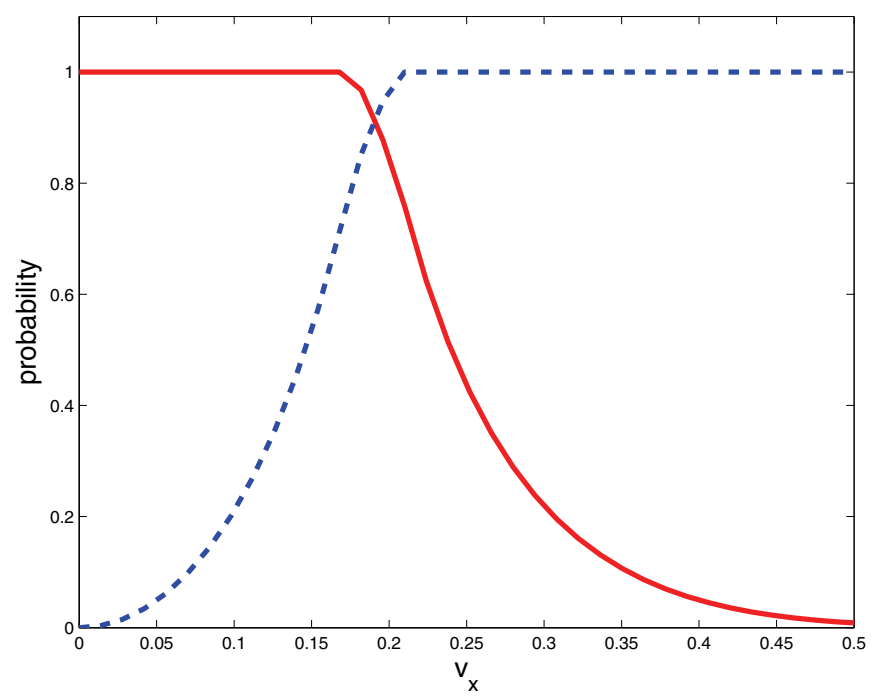

FIG. 1. Probabilities $p_{T}$ (solid line) and $p_{D}$ (dashed line) for thermalization and dethermalization as functions of $v=|\mathbf{v}|$, in which $v_{1}=0.1774$ and $v_{2}=0.2031$.

Equations (30) and (31) determine $\alpha$ for $|\mathbf{v}|<v_{1}$ (i.e., for $\gamma<\gamma_{1}$ ) and $|\mathbf{v}|>v_{2}$ (i.e., for $\gamma>\gamma_{2}$ ). Define $\alpha$ in the interval $\gamma_{1}<\gamma<\gamma_{2}$ by interpolation with respect to $\gamma$ to get

$$
\alpha= \begin{cases}(\gamma-1) & \text { for }|\mathbf{v}|<v_{1}, \\ \alpha_{1}+\frac{\left(\gamma-\gamma_{1}\right)}{\left(\gamma_{2}-\gamma_{1}\right)}\left(\alpha_{2}-\alpha_{1}\right) & \text { for } v_{1}<|\mathbf{v}|<v_{2}, \\ (\gamma-1)^{-1} & \text { for } v_{2}<|\mathbf{v}|\end{cases}
$$

Figure 1 shows a typical graph of the probabilities $p_{T}$ and $p_{D}$ as functions of $v$.

Note that the choice $c=1$, along with (27), determines the mass of the theoretically predicted Maxwellian component of $m=m_{t h}$ to be

$$
n_{m}=n_{M}\left(T_{m} / T_{M}\right)^{3 / 2} .
$$

In addition, the values of $p_{T}$ for $|\mathbf{v}|<v_{1}$ and $p_{D}$ for $v_{2}<|\mathbf{v}|$ could be set to values $\bar{p}_{T}$ and $\bar{p}_{D}$ that are different from 1 , and the formulas above could be modified to accommodate this change.

4.3. $s$-based method. In order to correctly incorporate the time step $\Delta t$ into the hybrid method, we base the thermalization/dethermalization probabilities $p_{T}$ and $p_{D}$ on Nanbu's parameter $s$ from (9) rather than v. Choose values of $s_{1}$ and $s_{2}$ with $s_{1}>s_{2}>0$. For each value of $\Delta t$, determine values of $v_{1}$ and $v_{2}$, so that $s\left(v_{1}, \Delta t\right)=s_{1}$ and $s\left(v_{2}, \Delta t\right)=s_{2}$. Then use the method in section 4.2 with these values of $v_{1}$ and $v_{2}$.

The choice of $p_{T}$ and $p_{D}$ described above is somewhat arbitrary; optimizing this choice subject to the condition (29) (or some improvement on this condition, as in Appendix B) could lead to an improved hybrid method.

5. Computational results. The computational results presented below are for bump-on-tail and Maxwellian initial data, as described in section 5.1. There are four sets of computations. Section 5.2 contains a set of consistency tests of the method. Computations illustrating the character of the solutions of the hybrid method for 
a bump on tail are presented in section 5.3. The effect of varying the numerical parameters $\Delta t, s_{1}$, and $s_{2}$ is shown in section 5.4. Finally, quantitative measurements of accuracy and efficiency of the hybrid method are presented in section 5.5.

5.1. Bump-on-tail and Maxwellian initial data. As a test of the hybrid method, we performed a series of computations for initial data that is a bump on tail. As discussed in section 1, this problem involves two widely separated time scales for Coulomb interactions, so that it is well suited for the hybrid method: a fast time scale for collisions between particles within the central Maxwellian and a slower time scale for those between particles from the central Maxwellian and the bump. We also performed computations for initial data that is Maxwellian, in order to test the consistency of the hybrid method.

The bump-on-tail initial distribution $f_{0}(\mathbf{v})$ is specified to be a combination of a Maxwellian $M_{0}(\mathbf{v})$ and a bump $g_{0}(\mathbf{v})$. The bump is specified to be approximately a $\delta$-function containing $10 \%$ of the mass of the distribution and centered at $\mathbf{v}=\left(v_{b}, 0,0\right)$ with $v_{b}=a \sqrt{T / m_{e}}$. The Maxwellian $M_{0}$ is centered and scaled, so that the average velocity of $f$ is 0 and the temperature is $T$. The examples presented here are for two different choices of $a: a=4$ in problem BOT4 and $a=3$ in problem BOT3.

The computations were performed in a dimensionless formulation in which the electron mass is $m_{e}=1$, and the electron density $n$ and temperature $T$ were chosen to be $n=0.1$ and $T=0.05065776$. For a characteristic time for the collision process, we use

$$
\begin{aligned}
t_{c} & =u_{t h}^{3}\left(n c_{F P}\right)^{-1}, \\
u_{t h} & =\sqrt{6 T / m_{e}},
\end{aligned}
$$

which has value $t_{c}=5.348275$. Unless otherwise stated, the number of particles is $N=128,000$. Note that in all of the simulation examples reported here, the plasma is spatially homogeneous, so that there are no electromagnetic fields and no convection.

5.2. Consistency tests. As a consistency test, we first performed computations for Maxwellian initial data $M(\mathbf{v})$, with density $n=0.1$, temperature $T=0.05065776$, and zero average velocity, as stated above.

Figure 2 shows the result of simulations using the hybrid method with this initial data for two different values of the hybrid parameters $s_{1}$ and $s_{2}$. The hybrid method parameters are $\left(s_{1}, s_{2}\right)=(2,1)$ on the left and $\left(s_{1}, s_{2}\right)=(1,0.5)$ on the right; the time step is $\Delta t=t_{c} / 100$. The total distribution $f=m+k$ (upper curves) and the thermal component $m$ (lower curves) of the distribution are shown as a function of the $x$-velocity $v_{x}$ at three times: $t=0, t=8.8 t_{c}, t=18.5 t_{c}$. The initial data consists of all particles; i.e., $k=M$ and $m=0$ for $t=0$. The total distribution $f$ is the same for all $t$, which is consistent with its Maxwellian initial data. Although it starts at 0 , by time $t=8.8 t_{c}$ (i.e., after an initial transient), the thermal component $m$ has reached a nonzero steady state which is the same as its value at $t=18.5 t_{c}$. This demonstrates the success of the detailed balance condition (29). Also shown is the theoretically predicted thermal component $m_{t h}$ from the choice $c=1$ for which the density is given by (27). Although the theoretical prediction is approximately correct for the hybrid simulation on the left, it is incorrect for the simulation on the right. A better theory (better than that of section 4.1) could help to improve the formulation of the hybrid method.

Next we perform a comparison of the $s$-based and $v$-based hybrid methods on the bump-on-tail problem BOT4. Set $\left(s_{1}, s_{2}\right)=(3,2)$ and $\Delta t=t_{c} / 10$. The corre- 

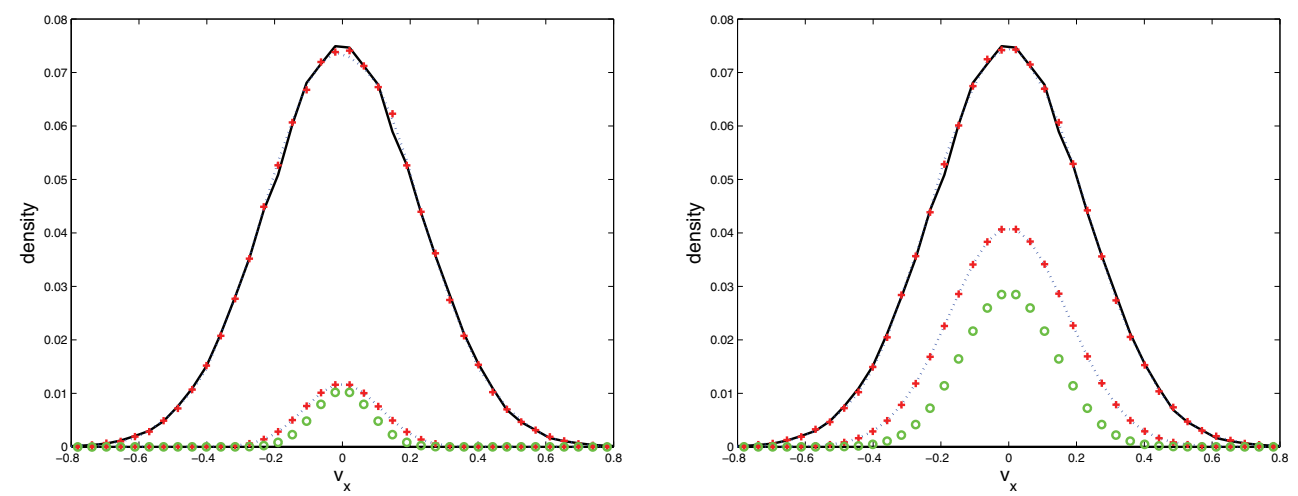

FIG. 2. Comparison of the hybrid method for Maxwellian (equilibrium) initial data at three different times: $t=0$ (solid line), $t=8.8 t_{c}$ (dotted line) and $t=18.5 t_{c}(+)$. The plots show the full distribution function $f\left(v_{x}\right)$ (upper curves) and the thermal component $m\left(v_{x}\right)$ (lower curves) at each of these times. The full distribution function is approximately the same at all times. The thermal component is 0 at $t=0$ but is equal for the two later times. Also shown is the thermal component (circles) predicted in (36) from sections 4.1 and 4.2. The hybrid method parameters are $\left(s_{1}, s_{2}\right)=(2,1)$ (left) and $\left(s_{1}, s_{2}\right)=(1,0.5)$ (right).

sponding values of $v_{1}$ and $v_{2}$, satisfying $s=S(v, \Delta t)$ defined by $(9)$, are $\left(v_{1}, v_{2}\right)=$ $(0.1774,0.2031)$. The top row of graphs in Figure 3 shows the results for the $v$-based method (upper left) and the $s$-based method (upper right) with these parameters. Each graph shows a comparison of results from the hybrid (dashed line) and Nanbu (solid line) methods at time $t=1.3 t_{c}$. These graphs are identical (and show good agreement between the hybrid and Nanbu methods), since the values of $\left(v_{1}, v_{2}\right)$ were chosen to be in agreement with the values of $\left(s_{1}, s_{2}\right)$.

Now keep the same values of $\left(v_{1}, v_{2}\right)$ and $\left(s_{1}, s_{2}\right)$ but change the time step to $\Delta t=t_{c} / 1000$. The results (on the lower graphs of Figure 3) show that the accuracy of the $v$-based method (lower left) deteriorates as the time step is decreased, whereas the accuracy of the $s$-based method (lower right) improves. In addition, the thermal component (dotted line) for the $s$-based method decreases with the smaller time step, so that the efficiency of the $s$-based method decreases. This gain in accuracy but loss of efficiency for the $s$-based method has acceptable dependence on $\Delta t$, while the loss of accuracy with decreased $\Delta t$ for the $v$-based method is not acceptable.

The reason for this dependence on time step is easily understood. For the $v$ based method, the probability of thermalization in each time step is independent of the size of $\Delta t$, so that for small $\Delta t$ the thermalization occurs too frequently. On the other hand, for the $s$-based method, the thermalization per time step decreases as $\Delta t$ decreases, and the function $s(\Delta t)$ has the correct dependence on $\Delta t$, as well as on density and temperature.

5.3. Simulation for the evolution of a bump on tail. Figures 4 and 5 show a comparison of the solutions computed by the hybrid (dashed line) and Nanbu (solid line) methods for bump-on-tail problems BOT4 and BOT3, respectively, at various times between the initial time and a final time $t_{\max }=7.2 t_{c}$. For the hybrid method the parameters are $\left(s_{1}, s_{2}\right)=(3,2)$ and $\Delta t=t_{c} / 10$. The thermal component of the hybrid representation (15) (dotted line) is also plotted. Both figures show very good agreement between the hybrid and Nanbu curves, providing a measure of validation for the hybrid method. 

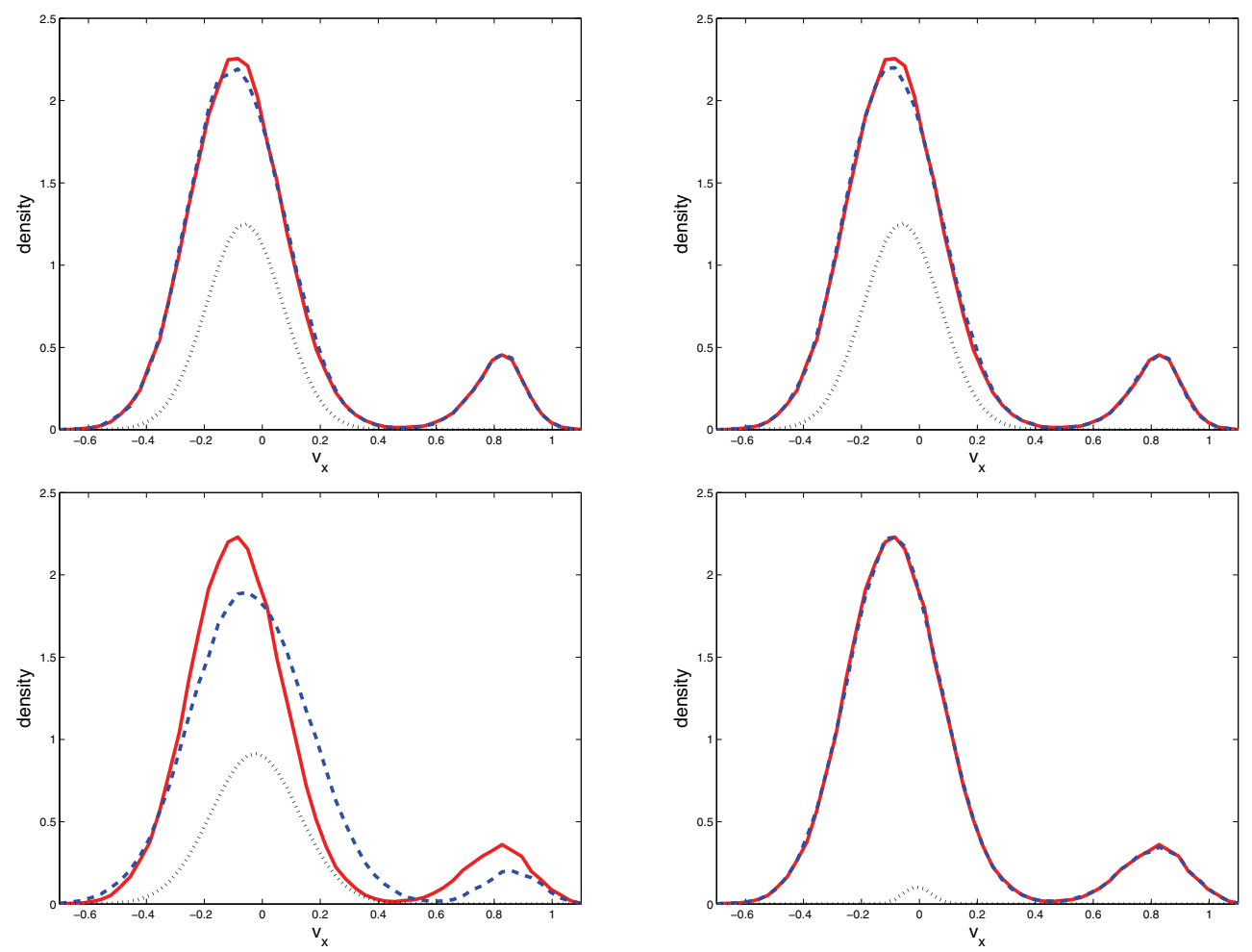

FIG. 3. Comparison of the v-based and s-based versions of the hybrid method for different time steps $\Delta t$. The plots show the velocity distribution function from the hybrid (dashed line) and Nanbu (solid line) methods, as well as the thermal component for the hybrid method (dotted line), for problem BOT4 at time $t=1.3 t_{c}$. The time step is $\Delta t=t_{c} / 10$ for the top row and $\Delta t=t_{c} / 1000$ for the bottom row. The left column comes from the $v$-based method with $\left(v_{1}, v_{2}\right)=(0.1774,0.2031)$, while the right column comes from the s-based method with $\left(s_{1}, s_{2}\right)=(3,2)$.

For problem BOT4 in Figure 4 the parameters are $\Delta t=t_{c} / 10$ and $\left(s_{1}, s_{2}\right)=(3,2)$. The thermal component of the hybrid representation (15) contains about $1 / 3$ of the particles.

For problem BOT3 in Figure 5 the parameters are $\Delta t=t_{c} / 100$ and $\left(s_{1}, s_{2}\right)=$ $(1,0.5)$. In this problem, the thermal component of the hybrid representation (15) contains about $1 / 7$ of the particles.

5.4. Variation of parameters $\Delta t, s_{1}$, and $s_{2}$. In order to understand the effect of the parameters $\Delta t, s_{1}$, and $s_{2}$ on the solution of the hybrid method, we performed computations for the bump-on-tail problem BOT4 of Figure 4 with different parameter values. Figures 6 and 7 show the solution of BOT4 at $t=1.2 t_{c}$ and $t=3.6 t_{c}$, respectively. In each figure, the time step is $\Delta t=t_{c} / 10$ for the graphs in the left column and $\Delta t=t_{c} / 100$ for those in the right column. Also, the values of $\left(s_{1}, s_{2}\right)$ are $\left(s_{1}, s_{2}\right)=(1,0.5)$ for the graphs in the top row, $\left(s_{1}, s_{2}\right)=(2,1)$ for the middle row, and $\left(s_{1}, s_{2}\right)=(3,2)$ for the bottom row.

In Figure 6 at an early time, the bump is still distinct but is shifted and diffused from its original position and shape. In Figure 7 at a later time, the bump is no longer a distinct peak but has been reduced to a shelf in the distribution function. Comparison of the figures shows that for larger $\Delta t$ or smaller $s_{1}$ and $s_{2}$ the bump 

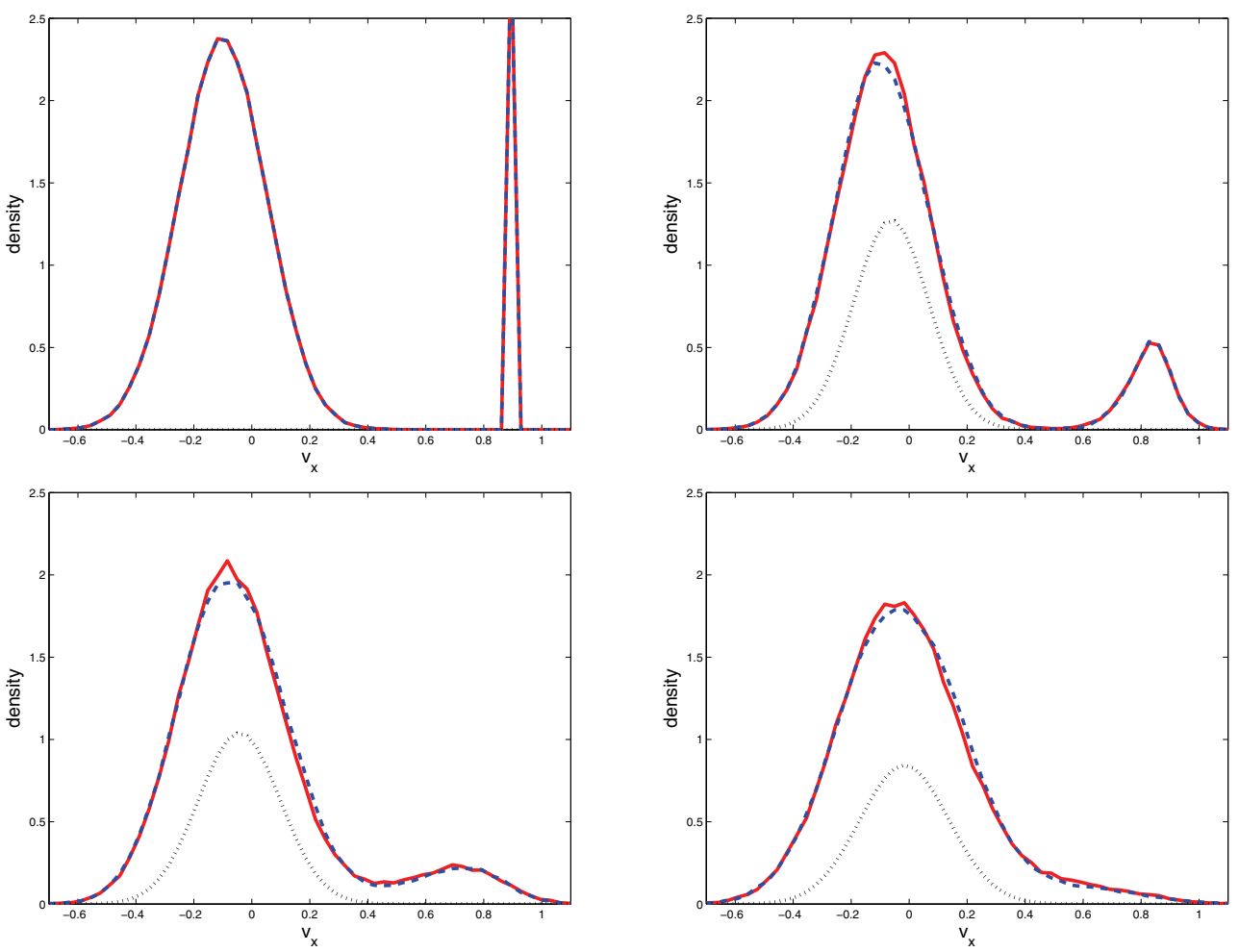

FiG. 4. Comparison of the hybrid (dashed line) and Nanbu (solid line) solutions, as well as the thermal component $m$ (dotted line) for the hybrid method, at different times $t=0$ (upper left), $t=1.2 t_{c}$ (upper right), $t=3.6 t_{c}$ (lower left), and $t=7.2 t_{c}$ (lower right). The computations use $\Delta t=t_{c} / 10$ and $\left(s_{1}, s_{2}\right)=(3,2)$ for problem BOT4.

is overthermalized, with the result that it is shifted too far toward the center and becomes too wide. As $\Delta t$ is decreased and $s_{1}$ and $s_{2}$ are increased, the accuracy of the computation dramatically improves. On the other hand, the size of the thermal component, which determines the efficiency of the hybrid method, is larger for larger values of $\Delta t$ and smaller values of $s_{1}$ and $s_{2}$. This shows a trade-off between efficiency and accuracy of the hybrid method.

5.5. Accuracy and efficiency for the hybrid method. First we present computations to illustrate the accuracy of the hybrid method for the problem BOT4. Figure 8 shows the fourth order moment of the velocity $\int|\mathbf{v}|^{4} f d \mathbf{v}$ and the anisotropy of the second order moments as functions of time for Nanbu's method and the hybrid method. For this problem, in which the initial perturbation of the Maxwellian is primarily in the $x$-velocity, the anisotropy is defined as $\int\left(v_{x}^{2}-\left(v_{y}^{2}+v_{z}^{2}\right)\right) f d \mathbf{v}$. For the same initial condition, Figure 9 shows the entropy decay (more precisely the decay of the $H$ function $\int f \log f d \mathbf{v}$ ) as a function of time for Nanbu's method and the hybrid method. These two figures show excellent agreement between the two methods for the anisotropy and entropy decay. The fourth order moment amplifies the differences of the two methods, especially in the bump, and has a difference of about $10 \%(0.0392$ for Nanbu's method and 0.0359 for the hybrid method) at the final time.

In order to measure the performance of the hybrid method, we first define quan- 

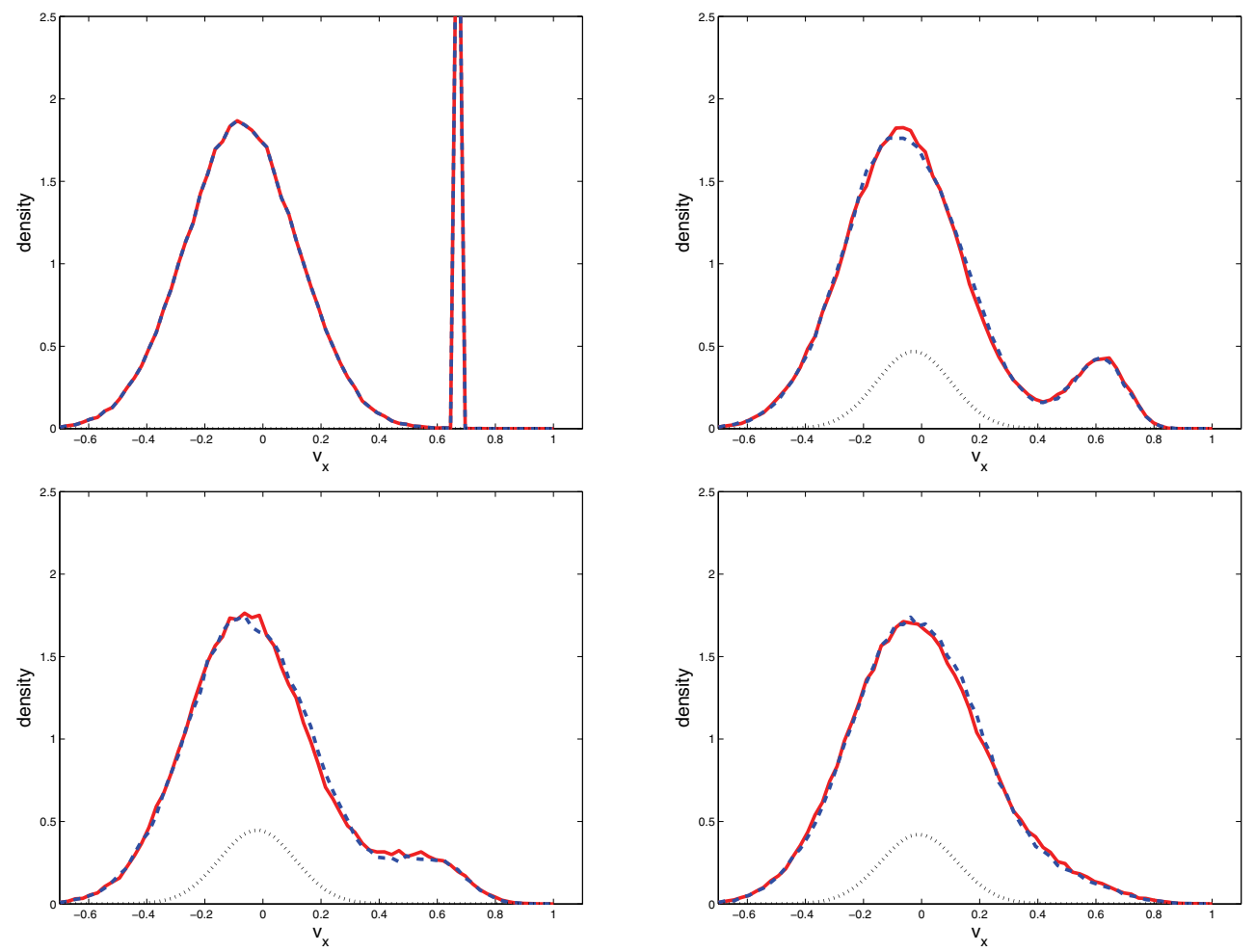

FiG. 5. Comparison of the hybrid (dashed line) and Nanbu (solid line) solutions, as well as the thermal component $m$ (dotted line) for the hybrid method, at different times $t=0$ (upper left), $t=0.6 t_{c}$ (upper right), $t=1.2 t_{c}$ (lower left), and $t=2.6 t_{c}$ (lower right). The computations use $\Delta t=t_{c} / 100$ and $\left(s_{1}, s_{2}\right)=(1,0.5)$ for problem BOT3.

tities $\gamma_{e f f}$ and $\gamma_{a c c}$ that measure the efficiency and accuracy of the computations as

$$
\begin{aligned}
\gamma_{e f f} & =\frac{1}{t_{\max } n_{f}} \int_{0}^{t_{\max }} n_{m} d t, \\
\gamma_{a c c} & =\frac{1}{t_{\max } n_{f}} \iint_{0}^{t_{\max }}\left|f-f_{H}\right| d t d v .
\end{aligned}
$$

Efficiency of the method is meant to be the ratio between the computational savings of the hybrid method and the computational cost of the standard method. Since the computational effort is roughly proportional to the number of particles in the simulation, the efficiency measure $\gamma_{e f f}$ is the ratio of $n_{m}$ and $n_{f}$, in which $n_{m}$ and $n_{f}$ are the number of particles in the Maxwellian component $m$ and the total number of particles in $f$. As a measure of accuracy, $\gamma_{a c c}$ is the relative size of $L^{1}$ norm (in $v$ and $t$ ) of the error.

We performed a series of computations for parameters in the range $0.2 \leq s_{2} \leq 2$ and $0.2 \leq s_{1}-s_{2} \leq 2$, as well as for time step $\Delta t=t_{c} / 10$ and final time $t_{\max }=74 t_{c}$. The resulting values of $\gamma_{e f f}$ and $\gamma_{a c c}$ are presented in contour plots in Figure 10, which shows them to be constant along (nearly) linear curves in the $\left(s_{1}, s_{2}\right)$ plane. A scatter plot of these values of $\gamma_{e f f}$ and $\gamma_{a c c}$ are presented in the graph on the left in Figure 11. This graph shows that these values collapse onto a single curve, so that 

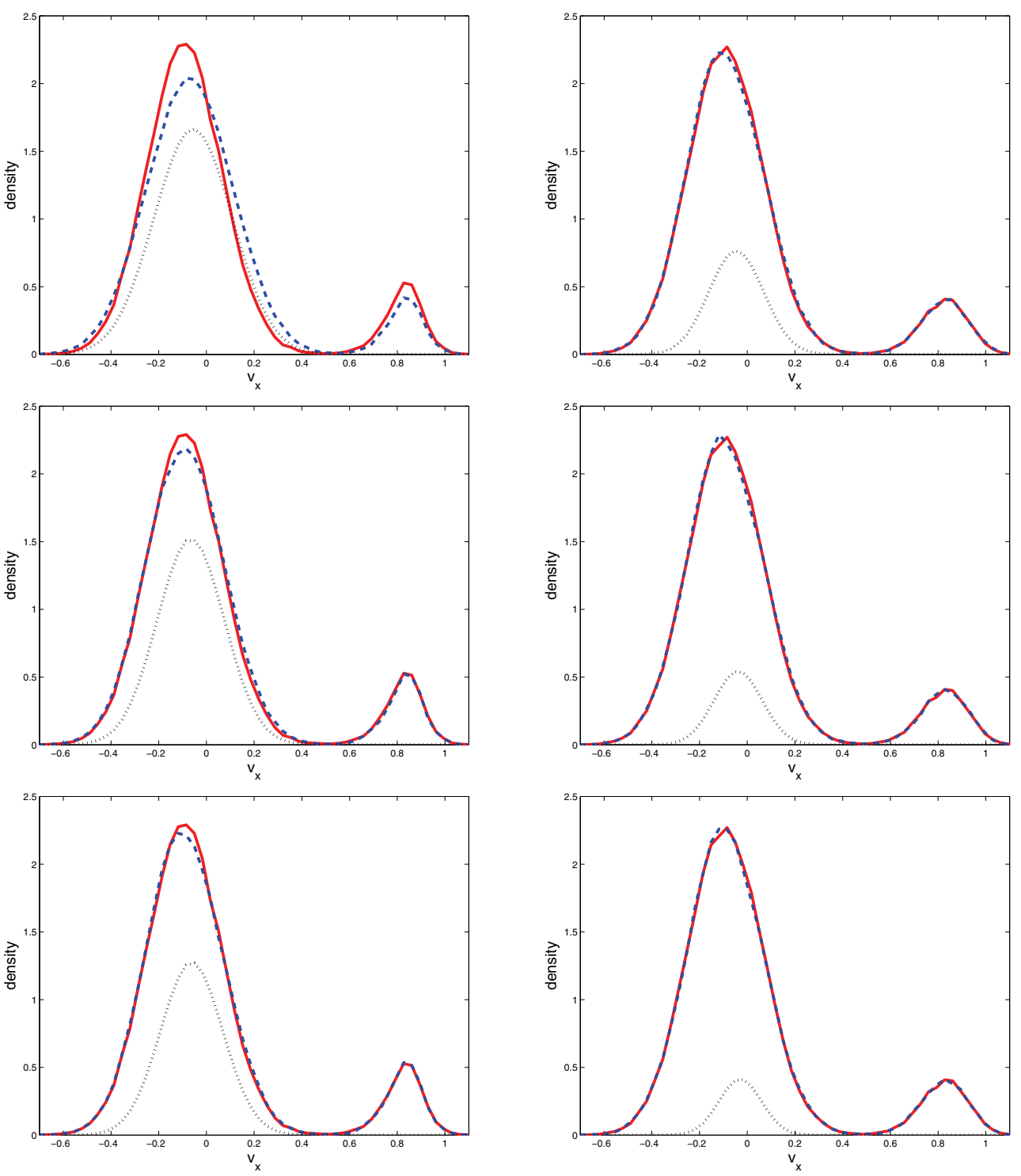

FIG. 6. Comparison of the hybrid (dashed line) and Nanbu (solid line) solutions, as well as the thermal component $m$ (dotted line) for the hybrid method, for different values of the parameters $\Delta t$, $s_{1}$, and $s_{2}$. The values of $\Delta t$ are $t_{c} / 10$ for the left column and $t_{c} / 100$ for the right column. The values of $\left(s_{1}, s_{2}\right)$ are $(1,0.5)$ for the top row, $(2,1)$ for the middle row, and $(3,2)$ for the bottom row. These simulations are for problem BOT4 of Figure 4 at time $t=1.2 t_{c}$.

$\gamma_{a c c}$ is a single-valued function of $\gamma_{e f f}$. This shows that for any level of accuracy there is a resulting level of efficiency. Further variation of the parameters $\left(s_{1}, s_{2}\right)$ does not change performance of the method. This conclusion holds only within the context of the specific choice of $p_{D}$ and $p_{T}$. The relationship between accuracy and efficiency could be changed by considering a wider class of functions $p_{D}$ and $p_{T}$.

In the graph on the left in Figure 11, the values of accuracy $\gamma_{a c c}$ appear to taper off to a finite nonzero value. The graph on the right in Figure 11 shows that 

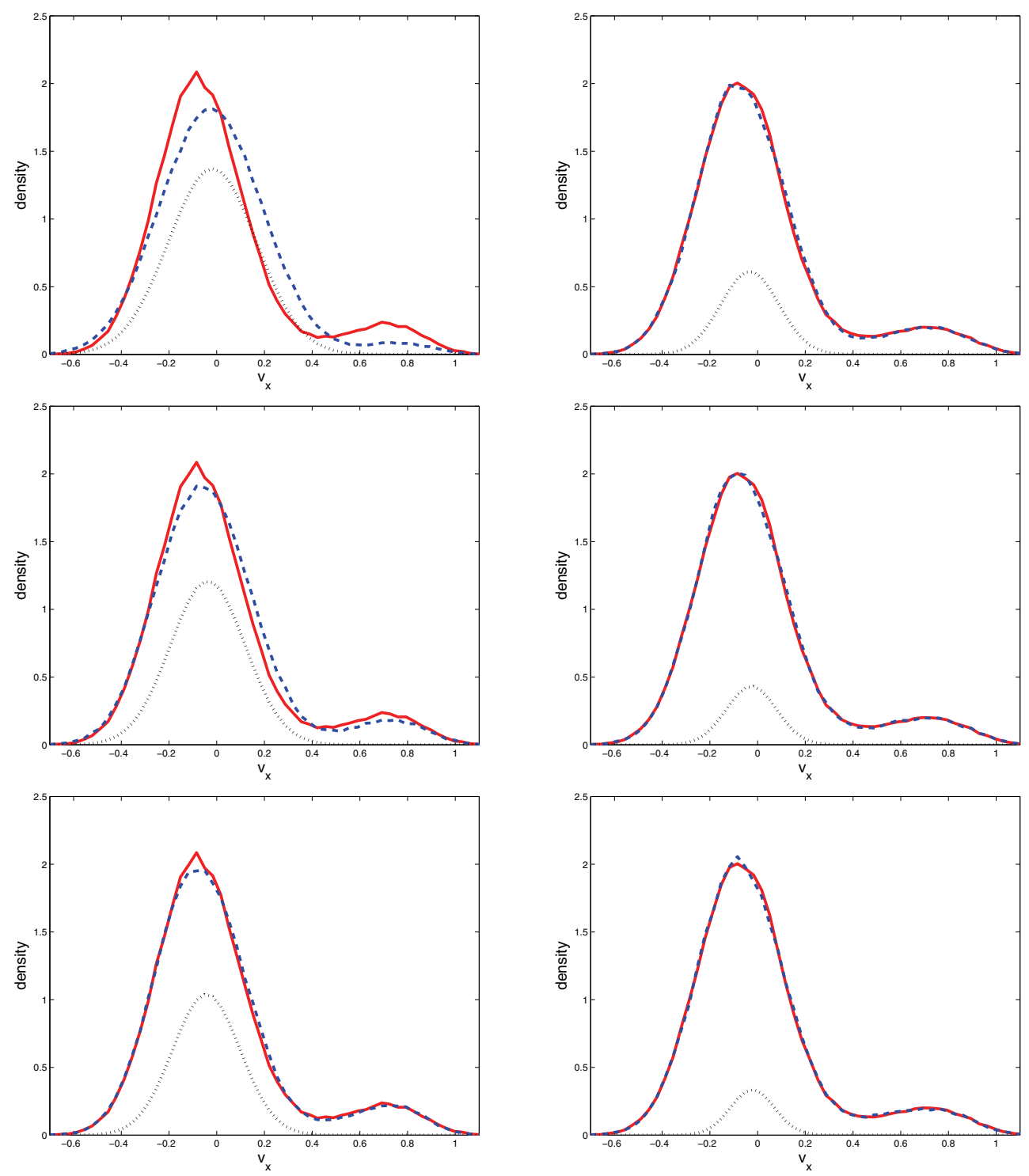

Fig. 7. Same as Figure 6 but at a later time: $t=3.6 t_{c}$.

statistical fluctuations due to the finite value $N$ of particles contribute importantly to this residual error. There may be an additional significant contribution to the total error due to finite $\Delta t$ effects. This graph shows a plot of $\gamma_{a c c}$ versus $\gamma_{e f f}$ for three values of $N: N=32,000, N=128,000$, and $N=512,000$. The values of $\left(s_{1}, s_{2}\right)$ are $4<s_{2}<6.2$ and $s_{1}=s_{2}+2$, which are larger than those in Figure 10 and the graph on the left of Figure 11.

Comparison of the results for $N=32,000, N=128,000$, and $N=512,000$ in this graph shows the errors $\gamma_{a c c}$ are smaller for larger values of $N$. More specifically, for larger values of $N$, the linear decrease of $\gamma_{a c c}$ continues for smaller values of $\gamma_{e f f}$, and the remaining residual value of $\gamma_{a c c}$ is smaller. 

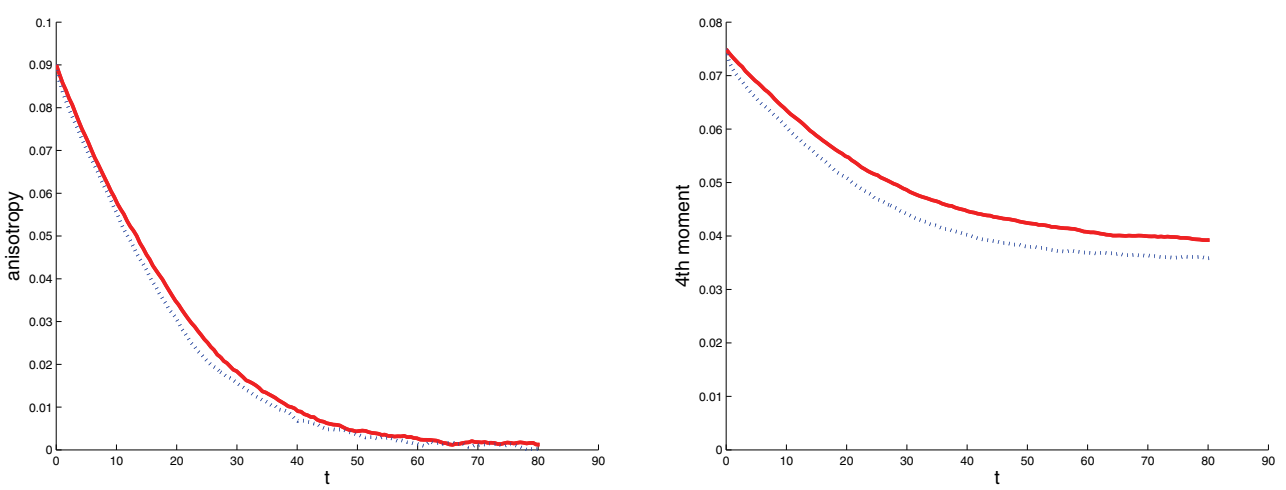

FIG. 8. Anisotropy of the second order moments (left) and fourth order moment (right) from the solutions of Nanbu (solid line) and the hybrid method (dotted line) for problem BOT4 of Figure 4.

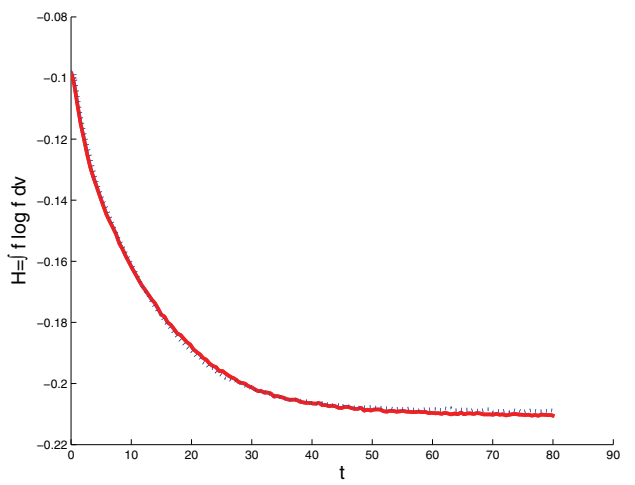

FIG. 9. The $H$ function from the solutions of Nanbu (solid line) and the hybrid method (dotted line) for problem BOT4 of Figure 4.
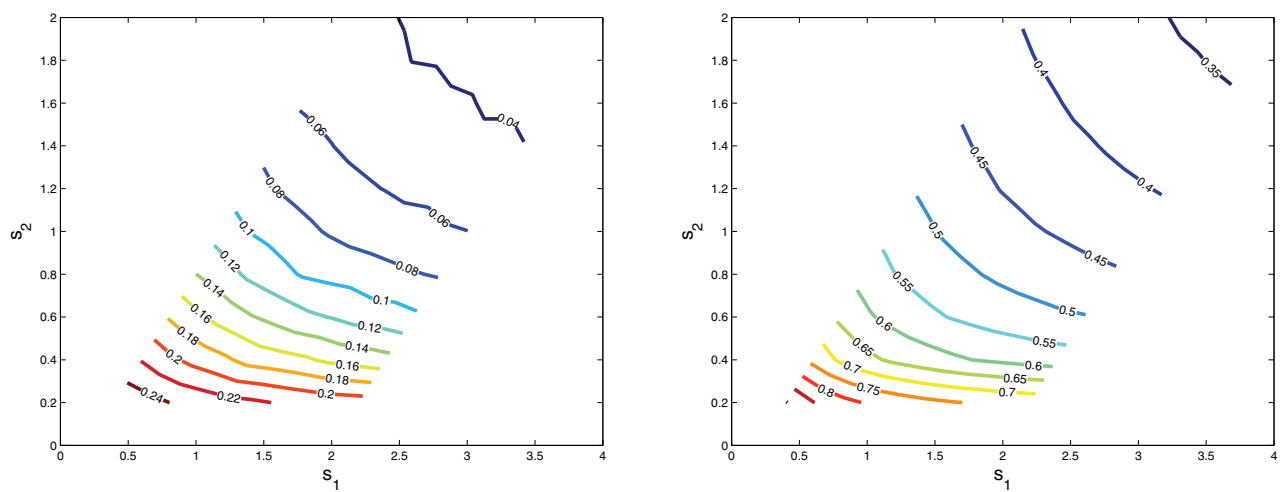

FIG. 10. Contour plots of error (left) and efficiency (right) for the hybrid method applied to problem BOT4 of Figure 4 with $N=128,000$ as functions of the parameters $s_{1}$ and $s_{2}$.

6. Conclusions. The hybrid method developed here combines continuum and particle descriptions for the evolution of a velocity distribution function through Coulomb interactions. The method includes particle interactions, but since the exam-

Copyright (c) by SIAM. Unauthorized reproduction of this article is prohibited. 

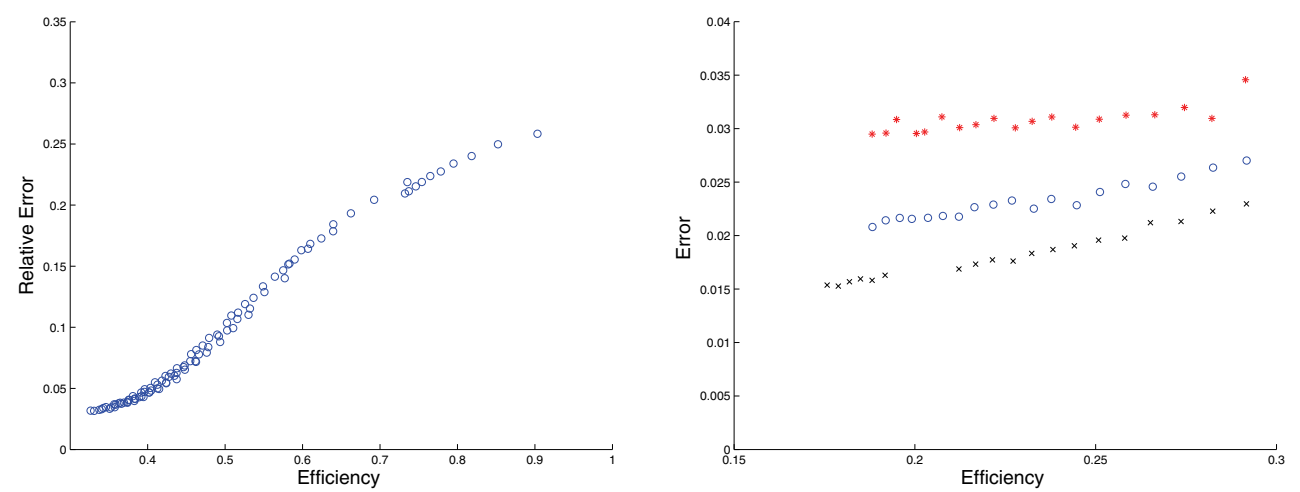

FIG. 11. Error versus efficiency for the bump-on-tail problem of Figure 4. The graph on the left is a scatter plot of the values of error and efficiency from Figure 10. The graph on the right shows a continuation of error and efficiency for larger values of $s_{1}$ and $s_{2}$ and for values of $N$ given by 32,000 (stars), 128,000 (circles), and 512,000 (x's).

ples here are spatially homogeneous, the continuum description is just an equilibrium Maxwellian distribution.

Because of the variation of the interaction rate as a function of particle velocity, the division of $f$ between particles and continuum must be performed as a function of velocity. In the hybrid method of this paper, the velocity dependence is effected through velocity dependence of the thermalization and dethermalization probabilities $p_{T}(v)$ and $p_{D}(v)$.

The specific choice of $p_{T}(v)$ and $p_{D}(v)$ is ad hoc and formulated in terms of two parameters $s_{1}$ and $s_{2}$ (or $v_{1}$ and $v_{2}$ ) as well as $\Delta t$. The simulations show that for this method the efficiency is a single-valued function of the accuracy of the method. Therefore the method provides a certain level of efficiency (acceleration) for prescribed accuracy of the hybrid approximation.

These results demonstrate the consistency and potential effectiveness of this hybrid approach for simulation of Coulomb collisions. Application of this method to real plasma physics problems will require progress in several directions: Further development of the hybrid method will hopefully make the method more efficient and accurate. Combination of the hybrid method with other methods, such as the drift/diffusion approach [8] or the $\delta f$ method $[3,4,5,6]$, could expand the range of application for the method. Particularly important is the need to include spatial inhomogeneity and particle advection in the hybrid method.

The efficiency of the hybrid method can be improved by decreasing the number of particles in the kinetic component. In contrast to the result in section 5.2 and Figure 2, for an optimal hybrid method the distribution would become completely thermalized as $t \rightarrow \infty$ (i.e., $m \rightarrow M$ and $k \rightarrow 0$ ) by increasing the size of the Maxwellian component $m$. We also plan to optimize the choice of the probabilities $p_{T}(v)$ and $p_{D}(v)$. Another possible generalization of the hybrid approach would include multiple Maxwellians in the hybrid representation of the velocity distribution function $f$. A mathematical foundation for this method is missing at present; its development, including an improved analysis of the detailed balance properties of the method, could be an important guide in improving the method. Our current work is now including the advection and acceleration of the particles, and we are replacing the "Maxwellian" particles with a fluid equation representation, which is when the real computational savings of the hybrid method is realized. 
Appendix A. Analysis of the scattering kernel $D$ for the TA method. In this appendix, we apply the convergence criteria of Bobylev and Nanbu [2] to show that the TA equation - (6) using the kernel $D_{T A}$ for TA — is a first order approximation of the Fokker-Planck equation.

The convergence criteria from equations (48a)-(48c) in [2] are that

$$
\begin{array}{r}
D(\mu, \tau) \geq 0, \\
2 \pi \int_{-1}^{1} d \mu D(\mu, \tau)=1, \\
\lim _{\tau \rightarrow 0} D(\mu, \tau)=(2 \pi)^{-1} \delta(1-\mu), \\
\lim _{\tau \rightarrow 0}(2 \pi / \tau) \int_{-1}^{1} d \mu D(\mu, \tau)\left[1-P_{\ell}(\mu)\right]=\ell(\ell+1),
\end{array}
$$

in which $P_{\ell}$ is the Legendre polynomial for positive integers $\ell$. Conditions (40) and (41) are reasonable restrictions that simplify the analysis; (42) is a consistency condition; and (43) is a first-order accuracy condition in terms of a Legendre polynomial expansion of the integral.

As written in (10), the kernel for the TA method is

$$
D_{T A}(\mu, \tau)=(2 \pi)^{-1}(2 \pi \tau)^{-1 / 2} e^{-\zeta^{2} / 2 \tau}(d \zeta / d \mu) .
$$

The analysis of this kernel is similar to the analysis of the kernel for the Nanbu method presented in [2]. Conditions (40)-(42) are easily verified.

To verify (43), use $\mu=\cos \theta$ and $\zeta=\tan (\theta / 2)$ to calculate for small $\tau$

$$
\begin{aligned}
& (2 \pi / \tau) \int_{-1}^{1} d \mu D_{T A}(\mu, \tau)\left[1-P_{\ell}(\mu)\right] \\
& \quad=\tau^{-1} \int_{0}^{\infty}(2 \pi \tau)^{-1 / 2} e^{-\zeta^{2} / 2 \tau}\left[1-P_{\ell}(\cos (2 \arctan \zeta))\right] d \zeta \\
& \quad=\tau^{-1} \int_{0}^{\infty}(2 \pi)^{-1 / 2} e^{-\xi^{2} / 2}\left[1-P_{\ell}(\cos (2 \arctan \sqrt{\tau} \xi))\right] d \xi \\
& \quad \approx \tau^{-1}(2 \pi)^{-1 / 2} \int_{0}^{\infty}(2 \pi)^{-1 / 2} e^{-\xi^{2} / 2} \tau \xi^{2} \ell(\ell+1) d \xi \\
& \quad \approx \ell(\ell+1) .
\end{aligned}
$$

These calculations use the expansion $P_{\ell}(\cos (2 \arctan \sqrt{\tau} \xi)) \approx 1-2 \tau \xi^{2} P_{\ell}^{\prime}(1)$ for small $\tau$ and $P_{\ell}^{\prime}(1)=\ell(\ell+1)$. This completes the verification of (40)-(43), which imply that the TA equation is a first order approximation of the Fokker-Planck equation.

Appendix B. Detailed balance for binary collisions with thermalization/dethermalization. For collisions between particles of a single species, (6) becomes

$$
f(\mathbf{v}, t+\Delta t)=\int_{R^{3} \times S^{2}} d \mathbf{w} d \mathbf{n} D\left(\frac{\mathbf{g} \cdot \mathbf{n}}{g}, \Lambda \frac{\Delta t}{g^{3}}\right) f\left(\mathbf{v}^{\prime}, t\right) f\left(\mathbf{w}^{\prime}, t\right) .
$$

Using the requirement [2] that $\int_{S^{2}} d \mathbf{n} D=1,(46)$ can be written as the following equation for the change $\Delta f$ in time $\Delta t$ :

$$
\Delta f(\mathbf{v})=\int_{R^{3} \times S^{2}} d \mathbf{w} d \mathbf{n} D\left(\frac{\mathbf{g} \cdot \mathbf{n}}{g}, \Lambda \frac{\Delta t}{g^{3}}\right)\left\{f\left(\mathbf{v}^{\prime}, t\right) f\left(\mathbf{w}^{\prime}, t\right)-f(\mathbf{v}, t) f(\mathbf{w}, t)\right\},
$$


which will be used in the formulation of detailed balance conditions.

Equation (46) can be rewritten to include thermalization and dethermalization. Since it is an equation for $f(\mathbf{v})$, the thermalization/dethermalization is applied only to the terms $f(\mathbf{v})$ and $f\left(\mathbf{v}^{\prime}\right)$ inside the integral. Using the representation $f=m+k$, the integral on the right-hand side of (46) becomes

$$
\begin{array}{r}
\int_{R^{3} \times S^{2}} d \mathbf{w} d \mathbf{n} D\left\{\left[m\left(\mathbf{v}^{\prime}\right) m\left(\mathbf{w}^{\prime}\right)+\left(1-p_{D}\right) m\left(\mathbf{v}^{\prime}\right) k\left(\mathbf{w}^{\prime}\right)+p_{T} k\left(\mathbf{v}^{\prime}\right) f\left(\mathbf{w}^{\prime}\right)\right]\right. \\
\left.+\left[p_{D} m\left(\mathbf{v}^{\prime}\right) k\left(\mathbf{w}^{\prime}\right)+\left(1-p_{T}\right) k\left(\mathbf{v}^{\prime}\right) f\left(\mathbf{w}^{\prime}\right)\right]\right\},
\end{array}
$$

in which

$$
D=D\left(\frac{\mathbf{g} \cdot \mathbf{n}}{g}, \Lambda \frac{\Delta t}{g^{3}}\right)
$$

Note that dethermalization is not applied to the term $m\left(\mathbf{v}^{\prime}\right) m\left(\mathbf{w}^{\prime}\right)$. In the integral (48) the velocities $\mathbf{v}^{\prime}$ and $\mathbf{w}^{\prime}$ are precollision values. In all of the formulas below, $p_{D}$ and $p_{T}$ are evaluated at $\mathbf{v}$, since the thermalization/dethermalization is applied after the collision. The terms in the first set of square brackets are the terms that contribute to the thermal component, while those in the second set of square brackets are the terms that contribute to the kinetic component. The contributions to the thermal component are projected onto a Maxwellian, so that

$$
\begin{aligned}
m(\mathbf{v}, t+\Delta t)= & \Pi_{M} \int_{R^{3} \times S^{2}} d \mathbf{w} d \mathbf{n} D\left[m\left(\mathbf{v}^{\prime}\right) m\left(\mathbf{w}^{\prime}\right)+\left(1-p_{D}\right) m\left(\mathbf{v}^{\prime}\right) k\left(\mathbf{w}^{\prime}\right)\right. \\
& \left.+p_{T} k\left(\mathbf{v}^{\prime}\right) f\left(\mathbf{w}^{\prime}\right)\right], \\
k(\mathbf{v}, t+\Delta t)= & \int_{R^{3} \times S^{2}} d \mathbf{w} d \mathbf{n} D\left[p_{D} m\left(\mathbf{v}^{\prime}\right) k\left(\mathbf{w}^{\prime}\right)+\left(1-p_{T}\right) k\left(\mathbf{v}^{\prime}\right) f\left(\mathbf{w}^{\prime}\right)\right] .
\end{aligned}
$$

The projection in (50) is equivalent to the following equations for $n_{m}, \mathbf{u}_{m}$, and $T_{m}$ :

$$
\begin{aligned}
\left(n_{m},\right. & \left.n_{m} \mathbf{u}_{m}, n_{m} T_{m}\right)(t+\Delta t) \\
= & \int_{R^{3} \times R^{3} \times S^{2}} d \mathbf{v} d \mathbf{w} d \mathbf{n} D\left(1, \mathbf{v},\left|\mathbf{v}-\mathbf{u}_{m}\right|^{2} / 2\right) \\
& \times\left[m\left(\mathbf{v}^{\prime}\right) m\left(\mathbf{w}^{\prime}\right)+\left(1-p_{D}\right) m\left(\mathbf{v}^{\prime}\right) k\left(\mathbf{w}^{\prime}\right)+p_{T} k\left(\mathbf{v}^{\prime}\right) f\left(\mathbf{w}^{\prime}\right)\right] .
\end{aligned}
$$

As in (47), these can be rewritten as equations for the changes in $k$ and in $n_{m}$, $\mathbf{u}_{m}$, and $T_{m}$; i.e.,

$$
\begin{aligned}
\left(\Delta n_{m}, \Delta\left(n_{m} \mathbf{u}_{m}\right), \Delta\left(n_{m} T_{m}\right)\right) & \\
= & \int_{R^{3} \times R^{3} \times S^{2}} d \mathbf{v} d \mathbf{w} d \mathbf{n} D\left(1, \mathbf{v},\left|\mathbf{v}-\mathbf{u}_{m}\right|^{2} / 2\right)\left[m\left(\mathbf{v}^{\prime}\right) m\left(\mathbf{w}^{\prime}\right)\right. \\
& \left.+\left(1-p_{D}\right) m\left(\mathbf{v}^{\prime}\right) k\left(\mathbf{w}^{\prime}\right)+p_{T} k\left(\mathbf{v}^{\prime}\right) f\left(\mathbf{w}^{\prime}\right)-m(\mathbf{v}) f(\mathbf{w})\right], \\
\Delta k(\mathbf{v})= & \int_{R^{3} \times S^{2}} d \mathbf{w} d \mathbf{n} D\left\{p_{D} m\left(\mathbf{v}^{\prime}\right) k\left(\mathbf{w}^{\prime}\right)+\left(1-p_{T}\right) k\left(\mathbf{v}^{\prime}\right) f\left(\mathbf{w}^{\prime}\right)-k(\mathbf{v}) f(\mathbf{w})\right\} .
\end{aligned}
$$

Copyright $\odot$ by SIAM. Unauthorized reproduction of this article is prohibited. 
The detailed balance condition says that in equilibrium $(f=m+k=M)$, each process and the reverse process exactly cancel. For (52) and (53), this says that

$$
\begin{aligned}
m\left(\mathbf{v}^{\prime}\right) m\left(\mathbf{w}^{\prime}\right)+\left(1-p_{D}\right) m\left(\mathbf{v}^{\prime}\right) k\left(\mathbf{w}^{\prime}\right)+p_{T} k\left(\mathbf{v}^{\prime}\right) f\left(\mathbf{w}^{\prime}\right) & =m(\mathbf{v}) f(\mathbf{w}), \\
p_{D} m\left(\mathbf{v}^{\prime}\right) k\left(\mathbf{w}^{\prime}\right)+\left(1-p_{T}\right) k\left(\mathbf{v}^{\prime}\right) f\left(\mathbf{w}^{\prime}\right) & =k(\mathbf{v}) f(\mathbf{w}) .
\end{aligned}
$$

Since $f=m+k=M$, the sum of (54) and (55) is just $M\left(\mathbf{v}^{\prime}\right) M\left(\mathbf{w}^{\prime}\right)=M(\mathbf{v}) M(\mathbf{w})$ for any collision pair $\left(\mathbf{v}^{\prime}, \mathbf{w}^{\prime}\right)$ before a collision and $(\mathbf{v}, \mathbf{w})$ after a collision, which is the usual detailed balance condition for the Maxwellian equilibrium. Equation (54) (or (55)) provides a single detailed balance condition on $p_{T}$ and $p_{D}$. Although it is not used in the hybrid method formulated above, the condition (54) may be useful for improving the current hybrid method. This condition is more complicated than the constraint (29) applied in section 4.1, and it may require that $p_{T}$ and $p_{D}$ depend on $\mathbf{v}^{\prime}, \mathbf{w}^{\prime}, \mathbf{v}$, and $\mathbf{w}$.

\section{REFERENCES}

[1] G. A. BIRD, Molecular Gas Dynamics and the Direct Simulation of Gas Flows, Oxford University Press, Oxford, UK, 1998.

[2] A. V. Bobylev And K. NAnBu, Theory of collision algorithms for gases and plasmas based on the Boltzmann equation and the Landau-Fokker-Planck equation, Phys. Rev. E (3), 61 (2000), pp. 4576-4582.

[3] A. M. Dimits And W. W. Lee, Partially linearized algorithms in gyrokinetic particle simulation, J. Comput. Phys., 107 (1993), pp. 309-323.

[4] A. M. Dimits, T. J. Williams, J. A. Byers, and B. I. Cohen, Scalings of ion-temperaturegradient-driven anomalous transport in tokamaks, Phys. Rev. Lett., 77 (1996), pp. 71-74.

[5] G. Hu and J. A. Krommes, Generalized weighting scheme for $f$ particle-simulation method, Phys. Plasmas, 1 (1994), pp. 863-874.

[6] M. Kotschenreuther, Numerical simulation, Bull. Am. Phys. Soc., 33 (1988), pp. 2107-2108.

[7] N. A. Krall And A. W. Trivelpiece, Principles of Plasma Physics, McGraw-Hill, New York, 1973.

[8] W. M. Mannheimer, M. Lampe, and G. Joyce, Langevin representation of Coulomb collisions in PIC simulations, J. Comput. Phys., 138 (1997), pp. 563-584.

[9] K. Nanbu, Theory of cumulative small-angle collisions in plasmas, Phys. Rev. E (3), 55 (1997), pp. 4642-4652.

[10] L. Pareschi and R. E. Caflisch, An implicit Monte Carlo method for rarefied gas dynamics, J. Comput. Phys., 154 (1999), pp. 90-116.

[11] M. N. Rosenbluth, W. M. MacDonald, and D. L. Judd, Fokker-Planck equation for an inverse-square force, Phys. Rev. (2), 107 (1957), pp. 1-6.

[12] M. Sherlock, A Monte-Carlo method for Coulomb collisions in hybrid plasma models, J. Comput. Phys., 227 (2008), pp. 2286-2292.

[13] L. Spitzer, Jr., Physics of Fully Ionized Gases, 2nd ed., Interscience, New York, 1967.

[14] T. TAKizuka AND H. ABE, A binary collision model for plasma simulation with a particle code, J. Comput. Phys., 25 (1977), pp. 205-219.

[15] B. A. Trubnikov, Particle interactions in a fully ionized plasma, in Reviews of Plasma Physics, Vol. 1, Consultant Bureau, New York, 1965, pp. 105-204.

[16] C. Wang, T. Lin, R. E. Caflisch, B. Cohen, and A. Dimits, Particle simulation of Coulomb collisions: Comparing the methods of Takizuka $\mathscr{B}$ Abe and Nanbu, J. Comput. Phys., 227 (2008), pp. 4308-4329.

Copyright (c) by SIAM. Unauthorized reproduction of this article is prohibited. 